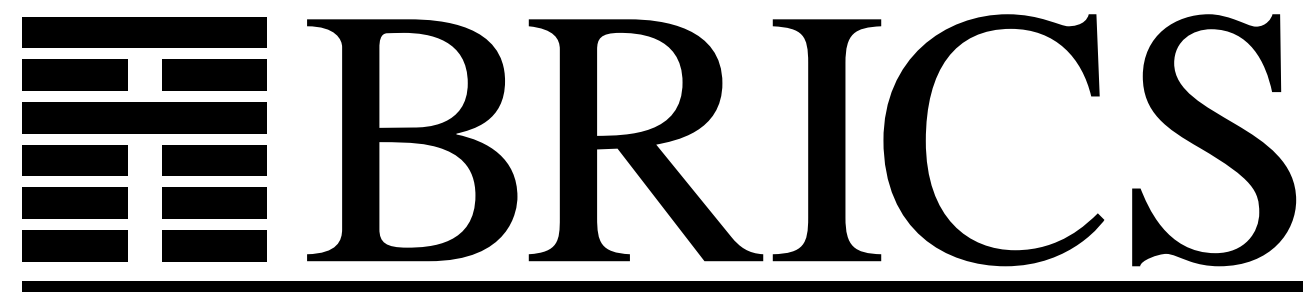

Basic Research in Computer Science

\title{
Precise Analysis of String Expressions
}

Aske Simon Christensen

Anders Møller

Michael I. Schwartzbach 
Copyright (c) 2003, Aske Simon Christensen \& Anders Møller \& Michael I. Schwartzbach.

BRICS, Department of Computer Science University of Aarhus. All rights reserved.

Reproduction of all or part of this work is permitted for educational or research use on condition that this copyright notice is included in any copy.

See back inner page for a list of recent BRICS Report Series publications. Copies may be obtained by contacting:

\author{
BRICS \\ Department of Computer Science \\ University of Aarhus \\ Ny Munkegade, building 540 \\ DK-8000 Aarhus C \\ Denmark \\ Telephone: +4589423360 \\ Telefax: $\quad+4589423255$ \\ Internet: BRICS@brics.dk
}

BRICS publications are in general accessible through the World Wide Web and anonymous FTP through these URLs:

http: //www.brics.dk

ftp: / / ftp.brics.dk

This document in subdirectory RS/03/5/ 


\title{
Precise Analysis of String Expressions
}

\author{
Aske Simon Christensen, Anders Møller, and Michael I. Schwartzbach \\ BRICS $^{\star \star}$, Department of Computer Science \\ University of Aarhus, Denmark \\ \{aske, amoeller,mis\}@brics.dk
}

\begin{abstract}
We perform static analysis of Java programs to answer a simple question: which values may occur as results of string expressions? The answers are summarized for each expression by a regular language that is guaranteed to contain all possible values. We present several applications of this analysis, including statically checking the syntax of dynamically generated expressions, such as SQL queries. Our analysis constructs flow graphs from class files and generates a context-free grammar with a nonterminal for each string expression. The language of this grammar is then widened into a regular language through a variant of an algorithm previously used for speech recognition. The collection of resulting regular languages is compactly represented as a special kind of multi-level automaton from which individual answers may be extracted. If a program error is detected, examples of invalid strings are automatically produced. We present extensive benchmarks demonstrating that the analysis is efficient and produces results of useful precision.
\end{abstract}

\section{Introduction}

To detect errors and perform optimizations in Java programs, it is useful to know which values that may occur as results of string expressions. The exact answer is of course undecidable, so we must settle for a conservative approximation. The answers we provide are summarized for each expression by a regular language that is guaranteed to contain all its possible values. Thus we use an upper approximation, which is what most client analyses will find useful.

This work is originally motivated by a desire to strengthen our previous static analysis of validity of dynamically generated XML documents in the JWIG extension of Java [4], but it has many other applications. Consider for example the following method, which dynamically generates an SQL query for a JDBC binding to a database:

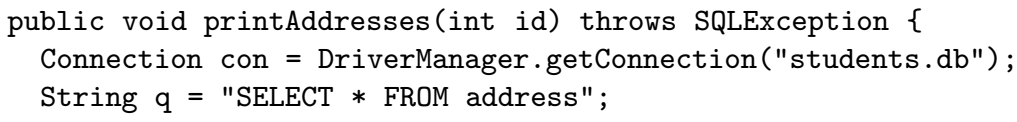

^ Supported by the Carlsberg Foundation contract number ANS-1069/20

** Basic Research in Computer Science (www.brics.dk), funded by the Danish National Research Foundation. 


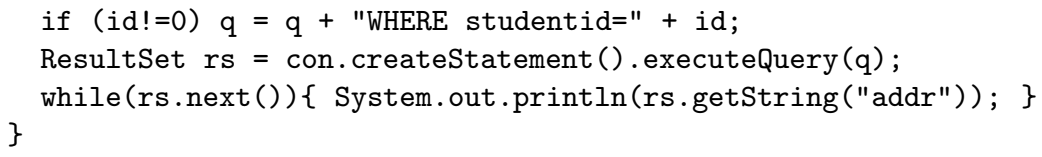

The query is built dynamically, so the compiler cannot guarantee that only syntactically legal queries will be generated. In fact, the above method compiles but the query will sometimes fail at runtime, since there is a missing space between address and WHERE. In general, it may range from tedious to difficult to perform manual syntax checking of dynamically generated queries.

Our string analysis makes such derived analyses possible by providing the required information about dynamically computed strings. We will use the term string operations when referring to methods in the standard Java library that return instances of the classes String or StringBuffer.

\section{Outline}

Our algorithm for string analysis can be split into two parts:

- a front-end that translates the given Java program into a flow graph, and

- a back-end that analyzes the flow graph and generates finite-state automata.

We consider the full Java language, which requires a considerable engineering effort. Translating a collection of class files into a sound flow graph is a laborious task involving several auxiliary static analyses. However, only the front-end is language dependent, hence the string analysis can be applied to other languages than Java by replacing just the front-end. The back-end proceeds in several phases:

- The starting point is the flow graph, which gives an abstract description of a program performing string manipulations. The graph only has def-use edges, thus control flow is abstracted away. Flow graph nodes represent operations on string variables, such as concatenation or substring.

- The flow graph is then translated into a context-free grammar with one nonterminal for each node. Flow edges and operations are modeled by appropriate productions. To boost precision, we use a special kind of grammar in which string operations are explicitly represented on right-hand sides.

- The context-free grammar is then transformed into a mixed left- and rightrecursive grammar using a variant of the Mohri-Nederhof algorithm [10], which has previously been used for speech recognition. The resulting grammar defines for each nonterminal the possible values of the string expression at the corresponding flow graph node.

- A program may contain many string expressions, but typically only few expressions, called hotspots, for which we actually want to know the regular language. For this reason, we introduce the multi-level automaton (MLFA), which is a compact data structure from which individual answers may be extracted by need. Extensive use of memoization helps to make these computations efficient. An MLFA is a well-founded hierarchical directed acyclic graph (DAG) of nondeterministic finite automata. 
All regular and context-free languages are over the Unicode alphabet, which we denote $\Sigma$. The core of the algorithm is the derivation of context-free grammars from programs and the adaptation of the Mohri-Nederhof algorithm [10], which provides an intelligent means of approximating a context-free language by a larger regular language. Naive solutions to this problem will not deliver sufficient precision in the analysis.

In programs manipulating strings, concatenation is the most important string operation - and in our analysis this operation is the one that we are able to model with the highest precision, since it is an inherent part of context-free grammars. We represent other string operations using less precise automata operations or character set approximations.

The translations from flow graph to multi-level automaton are all linear-time. The extraction of a deterministic finite-state automaton (DFA) for a particular string expression is worst-case doubly exponential: one for unfolding the DAG and one for determinizing and minimizing the resulting automaton. In the case of a monovariant analysis, the flow graph obtained from a Java program is in the worst case quadratic in the size of the program, but for typical programs, the translation is linear.

We provide a Java runtime library with operations for selecting the expressions that are hotspots, casting a string expression to the language of a specified regular expression, and for probing regular language membership. This library serves several purposes: 1) It makes it straightforward to apply our analysis tool. 2) In the same way normal casts affect type checking, this "regexp" cast operation can affect the string analysis since the casts may be assumed to succeed unless cast exceptions are thrown. This is useful in cases where the approximations made by the analysis are too rough, and it allows explicit specification of assertions about strings that originate from outside the analyzed program. 3) Even without applying the string analysis, the operations can detect errors, but at runtime instead of at compile-time.

In Section 2, we describe related work and alternative approaches. Section 3 defines flow graphs as the connection between the front-end and the back-end of the analysis. In Section 4, a notion of context-free grammars extended with operation productions is defined, and we show how to transform flow graphs into such grammars. Section 5 explains how a variant of the Mohri-Nederhof algorithm can be applied to approximate the grammars by strongly regular grammars. These are in Section 6 translated into MLFAs that efficiently allow minimal deterministic automata to be extracted for the hotspots of the original program. Section 7 sketches our implementation for Java, and Section 8 describes examples of string analysis applications and a number of practical experiments.

Due to the limited space, we omit proofs of correctness of the translation steps between the intermediate representations.

\section{Contributions}

The contributions in this paper consist of the following: 
- Formalization of the general framework for this problem and adaptation of the Mohri-Nederhof algorithm to provide solutions.

- Development of the MLFA data structure for compactly representing the resulting family of automata.

- A technique for delaying the approximation of special string operations to improve analysis precision.

- A complete open source implementation for the full Java language supporting the full Unicode alphabet.

- A Java runtime library for expressing regular language casts and checks.

- Experiments to demonstrate that the implementation is efficient and produces results of useful precision.

\section{Running Example}

In the following sections we illustrate the workings of the various phases of the algorithm on this tricky program:

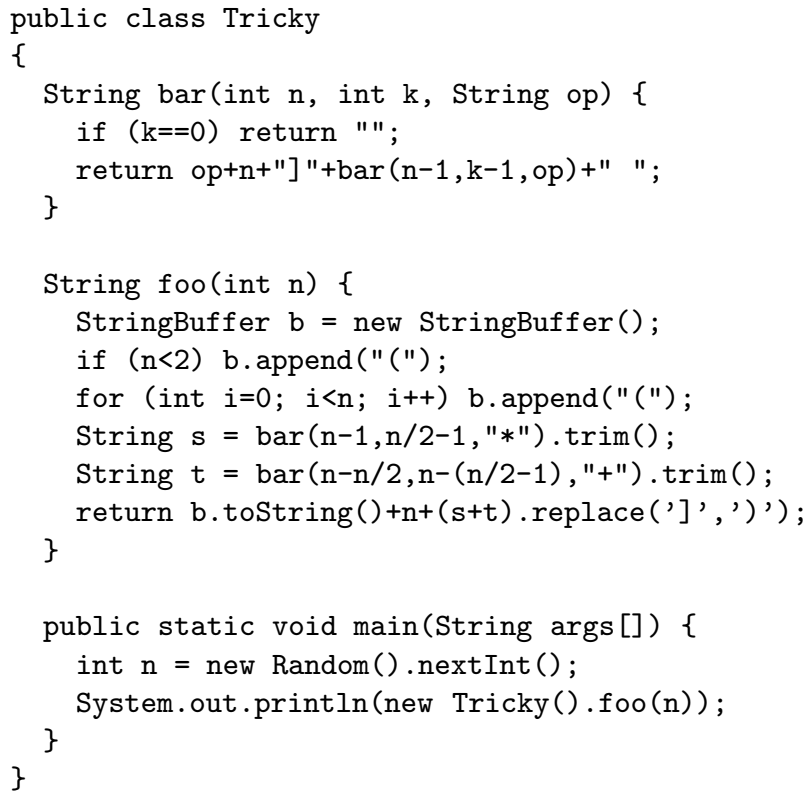

It computes strings of the form $((((((((8 * 7) * 6) * 5)+4)+3)+2)+1)+0)$ in a manner suitably convoluted to challenge our analysis.

\section{Related Work}

As far as we know, this straightforward problem of statically determining the possible values of string expressions has not really been explored before. We therefore choose to provide a discussion explaining why it cannot readily be solved using the standard techniques: abstract interpretation or set constraints. 
In both of those approaches, our work in obtaining a flow graph for string operations in Java programs would essentially have to be duplicated; the differences lie in the subsequent analysis of this flow graph.

Using the standard monotone framework for abstract interpretation [7, 12], the lattice of regular languages would be used to model abstract string values and all string operations would be given an abstract semantics. The standard fixedpoint iteration over the flow graph would, however, fail to provide a solution since the lattice of regular languages has infinite height. Thus, we would at some stage be required to perform a widening step. Finding an intelligent way of generalizing a regular language into a useful larger language becomes the stumbling block for this approach. Note that the context-free language defined by our derived grammar is in fact obtained as the fixed-point of a series of finite approximants. Thus, the Mohri-Nederhof algorithm may be viewed as a technique for jumping directly to a larger regular limit point.

Using set constraints [2], strings would be represented as linear terms with a constructor for each Unicode character. With this encoding, regular tree languages coincide with regular string languages. In the standard approach, each occurrence of an expression in the flow graph would be modeled by a set variable. String operations should then be modeled through appropriate set constraints on these variables. However, several of the operations we consider cannot be captured with any degree of precision by the permitted constraint operators. In particular, concatenation is not allowed: with such an operation, set constraints would no longer define regular tree languages [6]. Thus, we are returned to the problem that we solve in this paper: the flow graph inherently defines a contextfree language, which must subsequently be given a regular approximation.

A different approach is described in [15], which introduces the $\lambda^{r e}$-calculus where string expressions are typed by regular languages. This calculus allows in principle limited type inference (types of recursive functions must be given explicitly), but no algorithm is provided. Intriguingly, the paper refers to the Mohri-Nederhof algorithm as a possible venue for future work. In our approach, we use flow analysis rather than type inference. Thus, $\lambda^{r e}$ compares to our present work as XDuce [9] does to our previous work on JWIG [3].

There is of course much work in speech recognition related to the MohriNederhof algorithm, but we refer to their paper [10] for this discussion.

In our previous work on JWIG [4], we used a simple string analysis that keeps track of finite sets of strings but widens to $\Sigma^{*}$ at the slightest provocation. We believe that this simple algorithm has been used in many other places but has not been formally published.

Some work on machine learning is vaguely related to the problem we attack [13]: regular languages are inferred not from a flow graph but from a number of examples and answers to queries. We see no way of applying these techniques to our problem.

Other program analysis techniques also extract context-free grammars from programs [14], however, their grammars usually represent possible execution traces and never string values. Finally, we note that another well-known com- 
bination of strings and program analysis is unrelated to our work. In [8] the problem is to detect memory errors in manipulations of C-like string pointers, and the actual characters occurring in strings are irrelevant to the results.

\section{Definition of Flow Graphs}

A flow graph captures the flow of strings and string operations in a program while abstracting everything else away. The nodes in such a graph represent variables or expressions, and the edges are directed def-use edges that represent the possible data flow [1]. More precisely, a flow graph consists of a finite set $N$ of nodes of the following kinds:

- Init: construction of a string value, for instance from a constant or the Integer.toString method, and is associated a symbol reg that denotes a regular language $\llbracket r e g \rrbracket$.

- Join: an assignment or other join location.

- Concat: a string concatenation.

- UnaryOp: a unary string operation, for instance setCharAt or reverse, with an associated symbol $o p_{1}$ denoting a function $\llbracket o p_{1} \rrbracket: \Sigma^{*} \rightarrow \Sigma^{*}$. Non-string arguments to string operations are considered to be part of the function symbols.

- BinaryOp: a binary string operation, for instance insert, with an associated symbol $o p_{2}$ denoting a function $\llbracket o p_{2} \rrbracket: \Sigma^{*} \times \Sigma^{*} \rightarrow \Sigma^{*}$.

Init nodes have no incoming edges, Join nodes may have an arbitrary number of incoming edges, each UnaryOp node has exactly one incoming edge, and each Concat and BinaryOp node has an ordered pair of incoming edges that represent flow into the respective arguments. The flow graph for the Tricky example looks as follows:

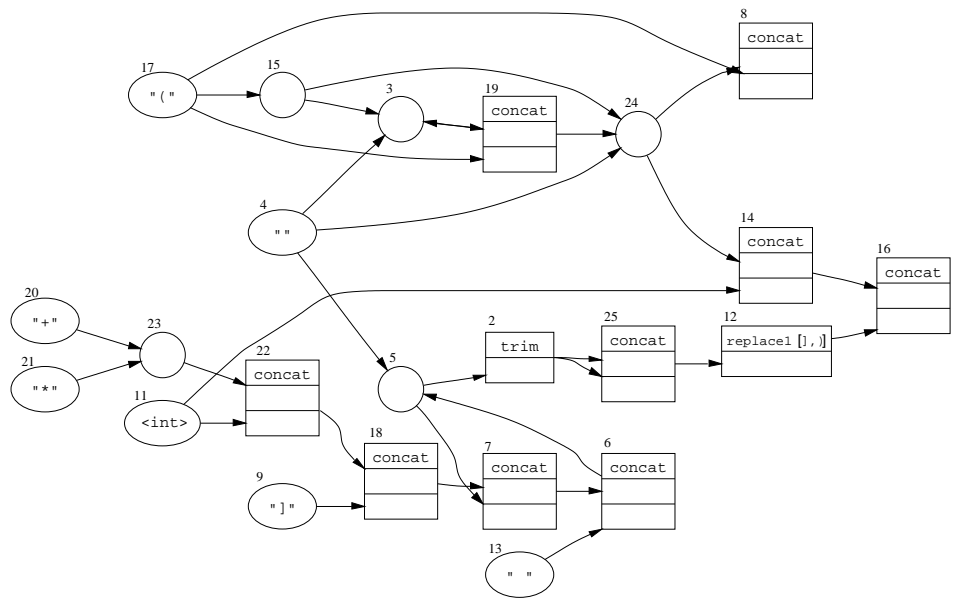

where the rightmost node corresponds to the single hotspot at println. The semantics of a flow graph can be defined as the least solution to a constraint 
system, similarly to the approach in [4]. The result is a map $m: N \rightarrow \Sigma^{*}$, such that $m(n)$ for every node $n$ contains all possible values of the expression or variable in the source program that corresponds to $n$.

\section{Construction of Context-Free Grammars}

From the flow graph, we construct a special context-free grammar such that each flow graph node $n \in N$ is associated a nonterminal $A_{n}$. This grammar has the following property: For each node $n$, the language $\mathcal{L}\left(A_{n}\right)$ of $A_{n}$ (that is, the language of the grammar with $A_{n}$ as start nonterminal) is the same as $m(n)$.

First, we define a context-free grammar with operation productions as a grammar where the productions are of the following kinds:

$$
\begin{array}{ll}
X \rightarrow Y & \text { [unit] } \\
X \rightarrow Y Z & \text { [pair] } \\
X \rightarrow r e g & \text { [regular] } \\
X \rightarrow o p_{1}(Y) & \text { [unary operation] } \\
X \rightarrow o p_{2}(Y, Z) & \text { [binary operation] }
\end{array}
$$

where $X, Y$, and $Z$ are nonterminals. The language of such a grammar is defined as one would expect: For a production $X \rightarrow$ reg, $X$ can derive all strings in $\llbracket r e g \rrbracket$. For a unary operation $X \rightarrow o p_{1}(Y), X$ can derive $\llbracket o p_{1} \rrbracket(\alpha)$ if $Y$ can derive $\alpha \in \Sigma^{*}$, and similarly for binary operations. Note that the language is not necessarily context-free because of the operation productions.

The translation from flow graphs to grammars is remarkably simple: For each node $n$, we add a nonterminal $A_{n}$ and a set of productions corresponding to the incoming edges of $n$ :

- For an Init node with language reg, add $A_{n} \rightarrow$ reg.

- For a Join node, add $A_{n} \rightarrow A_{m}$ for each node $m$ with an edge to $n$.

- For a Concat node, add $A_{n} \rightarrow A_{m} A_{p}$ where $m$ and $p$ are the two nodes that correspond to the pair of incoming edges of $n$.

- For a UnaryOp node with operation $o p_{1}$, add $A_{n} \rightarrow o p_{1}\left(A_{m}\right)$ where $m$ is the node having an edge to $n$.

- For a BinaryOp node with operation $o p_{2}$, add $A_{n} \rightarrow o p_{2}\left(A_{m}, A_{p}\right)$ where $m$ and $p$ are the two nodes that correspond to the pair of incoming edges of $n$.

The size of the resulting grammar is linear in the size of the flow graph. For the Tricky example it looks like:

$$
\begin{aligned}
& X_{2} \rightarrow \operatorname{trim}\left(X_{5}\right) \quad X_{3} \rightarrow X_{19} \quad X_{3} \rightarrow X_{15} \quad X_{3} \rightarrow X_{4} \\
& X_{4} \rightarrow " \text { " } \quad X_{5} \rightarrow X_{4} \quad X_{5} \rightarrow X_{6} \quad X_{6} \rightarrow X_{7} X_{13} \\
& \begin{array}{lllll}
X_{7} \rightarrow X_{18} X_{5} & X_{8} \rightarrow X_{24} X_{17} & X_{9} \rightarrow \text { "] " } & X_{11} \rightarrow \text { int }
\end{array} \\
& X_{12} \rightarrow \text { replace[], )](X25) } \quad X_{13} \rightarrow " \text { " } \quad X_{14} \rightarrow X_{24} X_{11} \quad X_{15} \rightarrow X_{17} \\
& X_{17} \rightarrow \text { "(" } \quad X_{16} \rightarrow X_{14} X_{12} \quad X_{18} \rightarrow X_{22} X_{9} \quad X_{19} \rightarrow X_{3} X_{17} \\
& X_{20} \rightarrow "+" \quad X_{21} \rightarrow " * n \quad X_{22} \rightarrow X_{23} X_{11} \quad X_{23} \rightarrow X_{20} \\
& X_{23} \rightarrow X_{21} \quad X_{24} \rightarrow X_{4} \quad X_{24} \rightarrow X_{15} \quad X_{24} \rightarrow X_{19}
\end{aligned}
$$

$X_{25} \rightarrow X_{2} X_{2}$

where the indices correspond to the node numbers in the flow graph. 


\section{Regular Approximation}

We wish to approximate the grammar generated in the previous section with a strongly regular grammar whose language contains that of the original.

As in the Mohri-Nederhof algorithm [10], we first find the strongly connected components of the grammar by viewing it as a graph with nonterminals as nodes and for each production an edge from the left-hand nonterminal to those on the right-hand side.

First, we eliminate all cycles that contain operation productions: For each unary operation $o p_{1}$ being used, we require a character set approximation $\llbracket o p_{1} \rrbracket_{C}$ : $2^{\Sigma} \rightarrow 2^{\Sigma}$ where $\llbracket o p_{1} \rrbracket_{C}(S)$ contains the set of characters that may occur in $\llbracket o p_{1} \rrbracket(x)$ for a string $x \in S^{*}$, and similarly for binary operations. Using these approximations in a simple fixed point computation on the grammar, we find for each nonterminal $X$ a set $C(X) \subseteq \Sigma$ containing all characters that may appear in the language of $X$. For each cycle we replace one operation production with $X \rightarrow r$ where $r$ denotes the regular language $C(X)^{*}$. After this transformation, the strongly connected components are recomputed. For the Tricky example, neither the trim nor the replace operation occurs on a cycle.

A component $M$ is right-generating if there exists a pair production $A \rightarrow B C$ such that $A$ and $B$ are in $M$, and $M$ is left-generating if there exists a pair production $A \rightarrow B C$ such that $A$ and $C$ are in $M$. Each component now has one of four types: simple if it is neither right- nor left-generating, left-linear if it is left-generating but not right-generating, right-linear if it is right-generating but not left-generating, and non-strongly-regular otherwise. The key observation of Mohri and Nederhof is that the desired approximation of the whole grammar can be obtained by a simple transformation of the non-strongly-regular components.

We adapt the Mohri-Nederhof algorithm to our form of grammar by transforming each non-strongly-regular component $M$ as follows: For each nonterminal $A$ in $M$, add a fresh nonterminal $A^{\prime}$. If $A$ corresponds to a hotspot or is used in another component than $M$, then add a production $A^{\prime} \rightarrow e$ where $e$ denotes $\{\epsilon\}$. Then replace all productions having $A$ as left-hand side as follows:

$$
\begin{array}{lll}
A \rightarrow X & \rightsquigarrow & A \rightarrow X A^{\prime} \\
A \rightarrow B & \rightsquigarrow & A \rightarrow B, \quad B^{\prime} \rightarrow A^{\prime} \\
A \rightarrow X Y & \rightsquigarrow & A \rightarrow R A^{\prime}, \quad R \rightarrow X Y \\
A \rightarrow X B & \rightsquigarrow & A \rightarrow X B, \quad B^{\prime} \rightarrow A^{\prime} \\
A \rightarrow B X & \rightsquigarrow & A \rightarrow B, \quad B^{\prime} \rightarrow X A^{\prime} \\
A \rightarrow B C & \rightsquigarrow & A \rightarrow B, \quad B^{\prime} \rightarrow C, \quad C^{\prime} \rightarrow A^{\prime} \\
A \rightarrow r e g & \rightsquigarrow & A \rightarrow R A^{\prime}, \quad R \rightarrow r e g \\
A \rightarrow o p_{1}(X) & \rightsquigarrow & A \rightarrow R A^{\prime}, \quad R \rightarrow o p_{1}(X) \\
A \rightarrow o p_{2}(X, Y) & \rightsquigarrow & A \rightarrow R A^{\prime}, \quad R \rightarrow o p_{2}(X, Y)
\end{array}
$$

Here, $A, B$, and $C$ are nonterminals within $M, X$ and $Y$ are nonterminals outside $M$, and each $R$ is a freshly generated nonterminal. Since all cycles with operation productions have been eliminated, the operation arguments cannot belong to $M$. As a result of this transformation, the component is now rightlinear, its size is proportional to the original one, and it is constructed in linear 
time. In contrast to Mohri and Nederhof's application where the grammar always has one fixed start nonterminal, our application requires regular approximation for all nonterminals that correspond to hotspots. Note that the language of a hotspot nonterminal in the original grammar is always a subset of the language of the same nonterminal in the approximated grammar.

We require for each unary operation $o p_{1}$ being used a conservative regular approximation (e.g. in the form of an automaton operation) $\llbracket o p_{1} \rrbracket_{R}: R E G \rightarrow$ $R E G$, where $R E G$ is the family of regular languages - and similarly for the binary operations. When the operations used in the grammar are replaced by their approximating counterparts, the language of each nonterminal is guaranteed to be regular.

The restriction on adding the $A^{\prime} \rightarrow e$ productions is essential for our application. As an example, consider the grammar:

$$
\begin{aligned}
& S \rightarrow T S \mid \mathrm{a} \\
& T \rightarrow S+
\end{aligned}
$$

which accepts strings of the form $a+a+\ldots+a$ and could be constructed from a tiny recursive Java method. Without the restriction, the resulting grammar would accept, for example, the string a+, which is an unacceptably rough approximation. Instead, the presented algorithm produces an approximation corresponding to the regular expression $\mathrm{a}(+\mathrm{a})^{*}$, which is the best we could hope for.

The Tricky example contains one non-strongly-regular component consisting of $\left\{X_{5}, X_{6}, X_{7}\right\}$, and the approximation algorithm transforms the grammar into the following:

$$
\begin{array}{llll}
X_{2} \rightarrow \operatorname{trim}\left(X_{5}\right) & X_{3} \rightarrow X_{19} & X_{3} \rightarrow X_{15} & X_{3} \rightarrow X_{4} \\
X_{4} \rightarrow " " & X_{5} \rightarrow X_{4} X_{5}^{\prime} & X_{5} \rightarrow X_{6} & X_{6}^{\prime} \rightarrow X_{5}^{\prime} \\
X_{6} \rightarrow X_{7} & X_{7}^{\prime} \rightarrow X_{13} X_{6}^{\prime} & X_{7} \rightarrow X_{18} X_{5} & X_{5}^{\prime} \rightarrow X_{7}^{\prime} \\
X_{8} \rightarrow X_{24} X_{17} & \left.X_{9} \rightarrow "\right] " & X_{11} \rightarrow<\text { int }> & \left.X_{12} \rightarrow \text { replace1[ , ) ](X } X_{25}\right) \\
X_{13} \rightarrow " " & X_{14} \rightarrow X_{24} X_{11} & X_{15} \rightarrow X_{17} & X_{16} \rightarrow X_{14} X_{12} \\
X_{17} \rightarrow "(" & X_{18} \rightarrow X_{22} X_{9} & X_{19} \rightarrow X_{3} X_{17} X_{20} \rightarrow "+" \\
X_{21} \rightarrow " * " & X_{22} \rightarrow X_{23} X_{11} & X_{23} \rightarrow X_{20} & X_{23} \rightarrow X_{21} \\
X_{24} \rightarrow X_{4} & X_{24} \rightarrow X_{15} & X_{24} \rightarrow X_{19} & X_{25} \rightarrow X_{2} X_{2} \\
X_{5}^{\prime} \rightarrow " " & & &
\end{array}
$$

with again $X_{16}$ corresponding to the hotspot.

\section{Multi-Level Finite Automata}

As in [10], we extract automata from strongly regular grammars. However, since we consider the language of more than one nonterminal and have the special operation productions, we use a novel formalism, multi-level finite automata (MLFA), with two important properties: 1) A strongly regular grammar can be translated into an equivalent MLFA in linear time, and 2) we can efficiently extract a minimal deterministic (normal) automaton for each hotspot. 
We define an MLFA to consist of a finite set of states $Q$ and a set of transitions $\delta \subseteq Q \times T \times Q$ where $T$ is a set of labels of the following kinds:

$$
\begin{aligned}
& \text { - reg } \\
& \text { - } \epsilon \\
& -(p, q) \\
& -o p_{1}(p, q) \\
& -o p_{2}\left(\left(p_{1}, q_{1}\right),\left(p_{2}, q_{2}\right)\right)
\end{aligned}
$$

where each $p$ and $q$ are states from $Q$. There must exist a level map $\ell: Q \rightarrow \mathbb{N}$ such that:

- $(s,(p, q), t) \in \delta \Rightarrow \ell(s)=\ell(t)>\ell(p)=\ell(q)$,

- $\left(s, o p_{1}(p, q), t\right) \in \delta \Rightarrow \ell(s)=\ell(t)>\ell(p)=\ell(q)$, and

- $\left(s, o p_{2}\left(\left(p_{1}, q_{1}\right),\left(p_{2}, q_{2}\right)\right), t\right) \in \delta \Rightarrow \ell(s)=\ell(t)>\ell\left(p_{i}\right)=\ell\left(q_{i}\right)$ for $i=1,2$.

That is, the states mentioned in a transition label are always at a lower level than the endpoints, and the endpoints are at the same level. The language $\overline{\mathcal{L}}$ of a single transition is defined according to its kind:

$$
\begin{aligned}
& \overline{\mathcal{L}}(r e g)=\llbracket r e g \rrbracket \\
& \overline{\mathcal{L}}(\epsilon)=\{\epsilon\} \\
& \overline{\mathcal{L}}((p, q))=\mathcal{L}(p, q) \\
& \overline{\mathcal{L}}\left(o p_{1}(p, q)\right)=\llbracket o p_{1} \rrbracket_{R}(\mathcal{L}(p, q)) \\
& \overline{\mathcal{L}}\left(o p_{2}\left(\left(p_{1}, q_{1}\right),\left(p_{2}, q_{2}\right)\right)\right)=\llbracket o p_{2} \rrbracket_{R}\left(\mathcal{L}\left(p_{1}, q_{1}\right), \mathcal{L}\left(p_{2}, q_{2}\right)\right)
\end{aligned}
$$

Let $\bar{\delta}(q, x)=\{p \in Q \mid(q, t, p) \in T \wedge x \in \overline{\mathcal{L}}(t)\}$ for $q \in Q$ and $x \in \Sigma^{*}$, and let $\widehat{\delta}: Q \times \Sigma^{*} \rightarrow 2^{Q}$ be defined by:

$$
\begin{aligned}
& \widehat{\delta}(q, \epsilon)=\bar{\delta}(q, \epsilon) \\
& \widehat{\delta}(q, x)=\{r \in Q \mid r \in \bar{\delta}(p, z) \wedge p \in \widehat{\delta}(q, y) \wedge x=y z \wedge z \neq \epsilon\} \text { for } x \neq \epsilon
\end{aligned}
$$

The language $\mathcal{L}(s, f)$ of a pair $s, f \in Q$ of start and final states where $\ell(s)=\ell(f)$ is defined as $\mathcal{L}(s, f)=\left\{x \in \Sigma^{*} \mid f \in \widehat{\delta}(s, x)\right\}$. This is well-defined because of the existence of the level map.

A strongly regular grammar produced in the previous section is transformed into an MLFA as follows: First, a state $q_{A}$ is constructed for each nonterminal $A$, and additionally, a state $q_{M}$ is constructed for each strongly connected component $M$. Then, for each component $M$, transitions are added according to the type of $M$ and the productions whose left-hand side are in $M$. For a simple or right-linear component:

$$
\begin{array}{lll}
A \rightarrow B & \rightsquigarrow & \left(q_{A}, \epsilon, q_{B}\right) \\
A \rightarrow X & \rightsquigarrow & \left(q_{A}, \Psi(X), q_{M}\right) \\
A \rightarrow X B & \rightsquigarrow & \left(q_{A}, \Psi(X), q_{B}\right) \\
A \rightarrow X Y & \rightsquigarrow & \left(q_{A}, \Psi(X), p\right),\left(p, \Psi(Y), q_{M}\right) \\
A \rightarrow r e g & \rightsquigarrow & \left(q_{A}, r e g, q_{M}\right) \\
A \rightarrow o p_{1}(X) & \rightsquigarrow & \left(q_{A}, o p_{1}(\Psi(X)), q_{M}\right) \\
A \rightarrow o p_{2}(X, Y) & \rightsquigarrow & \left(q_{A}, o p_{2}(\Psi(X), \Psi(Y)), q_{M}\right)
\end{array}
$$


For a left-linear component:

$$
\begin{array}{lll}
A \rightarrow B & \rightsquigarrow & \left(q_{B}, \epsilon, q_{A}\right) \\
A \rightarrow X & \rightsquigarrow & \left(q_{M}, \Psi(X), q_{A}\right) \\
A \rightarrow B X & \rightsquigarrow & \left(q_{B}, \Psi(X), q_{A}\right) \\
A \rightarrow X Y & \rightsquigarrow & \left(q_{M}, \Psi(X), p\right),\left(p, \Psi(Y), q_{A}\right) \\
A \rightarrow r e g & \rightsquigarrow & \left(q_{M}, r e g, q_{A}\right) \\
A \rightarrow o p_{1}(X) & \rightsquigarrow & \left(q_{M}, o p_{1}(\Psi(X)), q_{A}\right) \\
A \rightarrow o p_{2}(X, Y) & \rightsquigarrow & \left(q_{M}, o p_{2}(\Psi(X), \Psi(Y)), q_{A}\right)
\end{array}
$$

Each $p$ represents a fresh state. The function $\Psi$ maps each nonterminal into a state pair: If $A$ belongs to a simple or right-linear component $M$, then $\Psi(A)=$ $\left(q_{A}, q_{M}\right)$, and otherwise $\Psi(A)=\left(q_{M}, q_{A}\right)$. The construction is correct in the sense that the language of a nonterminal $A$ is equal to $\mathcal{L}(\Psi(A))$. We essentially follow Mohri and Nederhof, except that they construct an automaton for a fixed start nonterminal and do not have the unary and binary operations.

Given a hotspot from the source program, we find its flow graph node $n$, which in turn corresponds to a grammar nonterminal $A_{n}$ that is associated with a pair of states $(s, f)=\Psi\left(A_{n}\right)$ in an MLFA $F$. From this pair, we extract a normal nondeterministic automaton $U$ whose language is $\mathcal{L}(s, f)$ using the following algorithm:

- For each state $q$ in $F$ where $\ell(q)=\ell(s)$, construct a state $q^{\prime}$ in $U$. Let $s^{\prime}$ and $f^{\prime}$ be the start and final states, respectively.

- For each transition $\left(q_{1}, t, q_{2}\right)$ in $F$ where $\ell\left(q_{1}\right)=\ell\left(q_{2}\right)=\ell(s)$, add an equivalent sub-automaton from $q_{1}^{\prime}$ to $q_{2}^{\prime}$ : If $t=r e g$, we use a sub-automaton whose language is $\llbracket r e g \rrbracket$, and similarly for $t=\epsilon$. If $t=(p, q)$, then the sub-automaton is the one obtained by recursively applying the extraction algorithm for $\mathcal{L}(p, q)$. If $t=o p_{1}(p, q)$, the language of the sub-automaton is $\llbracket o p_{1} \rrbracket_{R}(\mathcal{L}(p, q))$, and similarly for $t=o p_{2}\left(\left(p_{1}, q_{1}\right),\left(p_{2}, q_{2}\right)\right)$.

This constructively shows that MLFAs define regular languages. The size of $U$ is worst-case exponential in the size of $F$ since the sub-automata are not reused. Since we subsequently determinize and minimize $U$, the size of the final DFA is worst-case doubly exponential, however, our experiments in Section 8 indicate that such blowups do not occur naturally. Our implementation uses memoization such that the automaton for a state pair $(s, f)$ is only computed once. This reuse of computations is important for programs with many hotspots.

We can now see the benefit of representing the unary and binary operations throughout all phases instead of, for instance, applying the character set approximation on all operations at an early stage: Those operations that in the flow graph do not occur in loops are modeled with higher precision than otherwise possible. For example, the insert method can be modeled quite precisely with an automaton operation, whereas that is difficult to achieve on the flow graph or grammar level. 


\section{Implementation for Java}

Our implementation works for the full Java language, which makes the translation to flow graphs quite involved and beyond the scope of this paper. Hence, we settle for a rough sketch.

We use the Soot framework [16] to parse class files and compute interprocedural control flow graphs. We give a precise treatment of String, StringBuffer, and multidimensional arrays of strings. Using a null-pointer analysis, we limit proliferation of null strings. The construction of the flow graphs further requires a constant analysis, a liveness analysis, a may-must alias analysis, and a reaching definitions analysis - all in interprocedural versions that conservatively take care of interaction with external classes.

Our analysis tool is straightforwardly integrated with client analyses, such as the ones described in the next section. Furthermore, it is connected to the runtime library mentioned in Section 1 such that regexp casts are fed into the analysis and the designated hotspots are checked.

\section{Applications and Experiments}

We have performed experiments with three different kinds of client analyses.

Our motivating example is to boost our previously published tool for analyzing dynamically generated XML in the JWIG extension of Java [4]. This tool uses a primitive string analysis as a black box that is readily upgraded to the one developed in this work.

Another example is motivated by the Soot framework [16] that we use in our implementation. Here a string analysis can be used to improve precision of call graphs for Java programs that use reflection through the Class.forName method.

Finally, it is possible to perform syntax checking of expressions that are dynamically generated as strings, as in the example in Section 1.

In all three cases we provide a number of benchmark programs ranging from small to medium sized. Each benchmark contains many string expressions, but only few of those are hotspots. For each benchmark we report the number of lines of Java code, the total number of string expressions, the number of hotspots considered, the number of seconds to compute the MLFA, the total number of seconds to provide automata for all hotspots, and the maximal memory consumption (in MB) during this computation. The timings do not include time used by Soot to load and parse the class files, which typically takes 5-10 seconds. The accuracy of the analysis is explained for each kind of application. All experiments are performed on a $1 \mathrm{GHz}$ Pentium III with 1 GB RAM running Linux.

\subsection{Tricky}

The Tricky benchmark is the example we followed in the previous sections, generating strings of the form: $(((((((8 * 7) * 6) * 5)+4)+3)+2)+1)+0)$. The analy- 
sis runs in 0.9 seconds and uses $26 \mathrm{MB}$ of memory. The regular approximation that we compute is (in Unix regexp notation) $\backslash(*<$ int $>([+*]<$ int $>\backslash)) *$ where <int> abbreviates $0 \mid(-$ ? [1-9] [0-9]*). This is a good result, but with a polyvariant analysis, the two calls to the bar method could be distinguished and the result further sharpened to $\backslash(*<$ int $>(\backslash *<$ int $>\backslash)) *(\backslash+<$ int $>\backslash)) *$.

\subsection{JWIG Validity Analysis}

The three smaller JWIG benchmarks are taken from the JWIG Web site. The four larger ones are an XML template manager where templates can be uploaded and edited (TempMan), a game management system (MyreKrig), a portal for a day care institution (Arendalsvej), and a system for management of the JAOO 2002 conference (JAOO). The hotspots correspond to places where strings are plugged into XML templates.

\begin{tabular}{|l|r|r|r|r|r|r|}
\hline Example & Lines & Exps & Hotspots & MLFA & Total & Memory \\
\hline Chat & 67 & 86 & 5 & 0.597 & 0.603 & 34 \\
\hline Guess & 77 & 50 & 4 & 0.577 & 0.581 & 34 \\
\hline Calendar & 89 & 116 & 6 & 0.712 & 0.828 & 34 \\
\hline Memory & 169 & 144 & 3 & 0.833 & 6.656 & 45 \\
\hline TempMan & 323 & 220 & 9 & 0.845 & 0.890 & 33 \\
\hline MyreKrig & 579 & 1,248 & 56 & 3.700 & 5.480 & 51 \\
\hline Arendalsvej & 3,725 & 5,517 & 274 & 20.767 & 35.473 & 102 \\
\hline JA00 & 3,764 & 9,655 & 279 & 39.721 & 86.276 & 107 \\
\hline
\end{tabular}

The time and memory consumptions are seen to be quite reasonable. The precision is perfect for these ordinary programs, where only URL syntax, integers and scalar values must be checked to conform to the requirements of XHTML 1.0. We use the DSD2 schema language [11] which is expressive enough to capture these requirements on string values. The string analysis typically takes $10-20 \%$ of the total JWIG analysis time.

\subsection{Reflection Analysis}

These benchmarks are culled from the Web by searching for programs that import java.lang.reflect and selecting non-constant uses of Class.forName which also constitute the hotspots.

\begin{tabular}{|l|r|r|r|r|r|r|}
\hline Example & Lines & Exps & Hotspots & MLFA & Total & Memory \\
\hline Switch & 21 & 45 & 1 & 1.155 & 1.338 & 25 \\
\hline ReflectTest & 50 & 95 & 2 & 1.117 & 1.220 & 25 \\
\hline SortAlgorithms & 54 & 31 & 1 & 0.997 & 1.214 & 25 \\
\hline CarShop & 56 & 30 & 2 & 0.637 & 0.656 & 25 \\
\hline ValueConverter & 1,718 & 438 & 4 & 4.065 & 4.127 & 36 \\
\hline ProdConsApp & 3,496 & 1,909 & 3 & 12.160 & 13.469 & 80 \\
\hline
\end{tabular}

Again, the time and memory consumptions are unremarkable. Without a client analysis, it is difficult to rate the precision. In simple cases like SortAlgorithms and CarShop we find the exact classes, and in some like ValueConverter we fail because strings originate from external sources. 


\subsection{Syntax Analysis}

Many Java programs build string expressions that are externally interpreted, a typical example being SQL queries handled by JDBC, as in Section 1. At present, no static syntax checking is performed on such expressions, which is a potential source of runtime errors. We can perform such checking by approximating the allowed syntax by a regular subset which is then checked to be a superset of the inferred set of strings. For SQL, we have constructed a regular language that covers most common queries and translates into a DFA with 631 states.

The benchmarks below are again obtained from the Web. Most originate from database textbooks or instruction manuals for various JDBC bindings. The hotspots correspond to calls of executeQuery and similar methods.

\begin{tabular}{|l|r|r|r|r|r|r|r|r|}
\hline Example & Lines & Exps & Hotspots & MLFA & Total & Memory & Errors & False Errors \\
\hline Decades & 26 & 63 & 1 & 0.669 & 1.344 & 27 & 0 & 0 \\
\hline SelectFromPer & 51 & 50 & 1 & 1.442 & 1.480 & 27 & 0 & 0 \\
\hline LoadDriver & 78 & 154 & 1 & 0.942 & 0.981 & 28 & 0 & 0 \\
\hline DB2Appl & 105 & 59 & 2 & 0.736 & 0.784 & 27 & 0 & 0 \\
\hline AxionExample & 162 & 37 & 7 & 0.800 & 1.008 & 29 & 0 & 0 \\
\hline Sample & 178 & 157 & 4 & 0.804 & 1.261 & 28 & 0 & 0 \\
\hline GuestBookServlet & 344 & 320 & 4 & 1.741 & 3.167 & 33 & 1 & 0 \\
\hline DBTest & 384 & 412 & 5 & 1.688 & 2.387 & 31 & 1 & 0 \\
\hline CoercionTest & 591 & 1,133 & 4 & 4.457 & 5.664 & 42 & 0 & 0 \\
\hline
\end{tabular}

As before, the analysis runs efficiently. We accept all the correct syntax, and encouragingly we find actual errors. The GuestBookServlet builds a string value with the construction " " + email + ")", where email is read directly from an input field in a Web form. Our tool responds by automatically generating the shortest counterexample:

INSERT INTO comments (id, email, name, comment, date) VALUES (0,', , , , ', ,')

which in fact points to a severe security flaw.

XPath expressions [5] are other examples where static syntax checking is desirable. Also, arguments to the method Runtime.exec could be checked to belong to a permitted subset of shell commands.

\section{Conclusion}

We have presented a static analysis technique for extracting a context-free grammar from a program and apply a variant of the Mohri-Nederhof approximation algorithm to approximate the possible values of string expressions in Java programs. The potential applications include validity checking of dynamically generated XML, improved precision of call graphs for Java programs that use reflection, and syntax analysis of dynamically generated SQL expressions.

Our experiments show that the approach is efficient and produces results of useful precision on realistic benchmarks. The open source implementation together with documentation and all benchmark programs are available at http://www.brics.dk/JSA/. 


\section{References}

[1] Alfred V. Aho, Ravi Sethi, and Jeffrey D. Ullman. Compilers - Principles, Techniques, and Tools. Addison-Wesley, November 1985.

[2] Alex Aiken. Introduction to set constraint-based program analysis. Science of Computer Programming, 35:79-111, 1999.

[3] Aske Simon Christensen, Anders Møller, and Michael I. Schwartzbach. Static analysis for dynamic XML. Technical Report RS-02-24, BRICS, May 2002. Presented at Programming Language Technologies for XML, PLAN-X, October 2002.

[4] Aske Simon Christensen, Anders Møller, and Michael I. Schwartzbach. Extending Java for high-level Web service construction. ACM Transactions on Programming Languages and Systems, 2003. To appear.

[5] James Clark and Steve DeRose. XML path language, November 1999. W3C Recommendation. http://www.w3.org/TR/xpath.

[6] H. Comon, M. Dauchet, R. Gilleron, F. Jacquemard, D. Lugiez, S. Tison, and M. Tommasi. Tree automata techniques and applications, 1999. Available from http://www.grappa.univ-lille3.fr/tata/.

[7] Patrick Cousot and Radhia Cousot. Abstract interpretation: a unified lattice model for static analysis of programs by construction or approximation of fixpoints. In Proc. 4th ACM SIGPLAN-SIGACT Symposium on Principles of Programming Languages, POPL 'r7, pages 238-252, 1977.

[8] Nurit Dor, Michael Rodeh, and Mooly Sagiv. Cleanness checking of string manipulations in C programs via integer analysis. In Proc. 8th International Static Analysis Symposium, SAS '01, volume 2126 of LNCS. Springer-Verlag, July 2001.

[9] Haruo Hosoya and Benjamin C. Pierce. XDuce: A typed XML processing language. In Proc. 3rd International Workshop on the World Wide Web and Databases, WebDB '00, volume 1997 of LNCS. Springer-Verlag, May 2000.

[10] Mehryar Mohri and Mark-Jan Nederhof. Robustness in Language and Speech Technology, chapter 9: Regular Approximation of Context-Free Grammars through Transformation. Kluwer Academic Publishers, 2001.

[11] Anders Møller. Document Structure Description 2.0, October 2002. BRICS, Department of Computer Science, University of Aarhus, Available from http://www.brics.dk/DSD/.

[12] Flemming Nielson, Hanne Riis Nielson, and Chris Hankin. Principles of Program Analysis. Springer-Verlag, October 1999.

[13] Rajesh Parekh and Vasant Honavar. DFA learning from simple examples. Machine Learning, 44:9-35, 2001.

[14] Thomas Reps. Program analysis via graph reachability. Information and Software Technology, 40(11-12):701-726, November/December 1998.

[15] Naoshi Tabuchi, Eijiro Sumii, and Akinori Yonezawa. Regular expression types for strings in a text processing language. In Proc. Workshop on Types in Programming, TIP '02, 2002.

[16] Raja Vallee-Rai, Laurie Hendren, Vijay Sundaresan, Patrick Lam, Etienne Gagnon, and Phong Co. Soot - a Java optimization framework. In Proc. IBM Centre for Advanced Studies Conference, CASCON '99. IBM, November 1999. 


\section{Recent BRICS Report Series Publications}

RS-03-5 Aske Simon Christensen, Anders Møller, and Michael I. Schwartzbach. Precise Analysis of String Expressions. February 2003. 15 pp.

RS-03-4 Marco Carbone and Mogens Nielsen. Towards a Formal Model for Trust. January 2003.

RS-03-3 Claude Crépeau, Paul Dumais, Dominic Mayers, and Louis Salvail. On the Computational Collapse of Quantum Information. January 2003. 31 pp.

RS-03-2 Olivier Danvy and Pablo E. Martínez López. Tagging, Encoding, and Jones Optimality. January 2003. To appear in Degano, editor, Programming Languages and Systems: Twelfth European Symposium on Programming, ESOP '03 Proceedings, LNCS, 2003.

RS-03-1 Vladimiro Sassone and Pawel Sobocinski. Deriving Bisimulation Congruences: 2-Categories vs. Precategories. January 2003. To appear in Gordon, editor, Foundations of Software Science and Computation Structures, FoSSaCS '03 Proceedings, LNCS, 2003.

RS-02-52 Olivier Danvy. A New One-Pass Transformation into Monadic Normal Form. December 2002. 16 pp. To appear in Hedin, editor, Compiler Construction, 12th International Conference, CC '03 Proceedings, LNCS, 2003.

RS-02-51 Gerth Stølting Brodal, Rolf Fagerberg, Anna Östlin, Christian N. S. Pedersen, and S. Srinivasa Rao. Computing Refined Buneman Trees in Cubic Time. December 2002. 14 pp.

RS-02-50 Kristoffer Arnsfelt Hansen, Peter Bro Miltersen, and V. Vinay. Circuits on Cylinders. December 2002. 16 pp.

RS-02-49 Mikkel Nygaard and Glynn Winskel. HOPLA-A HigherOrder Process Language. December 2002. 18 pp. Appears in Brim, Jančar, Křetínský and Antonín, editors, Concurrency Theory: 13th International Conference, CONCUR '02 Proceedings, LNCS 2421, 2002, pages 434-448. 